\title{
Radar track segmentation with cubic splines for collision risk models in high density terminal areas
}

\author{
JA Cózar', FJ Sáez ${ }^{2}$ and E Ricaud ${ }^{2}$
}

\begin{abstract}
This paper presents a method to segment airplane radar tracks in high density terminal areas where the air traffic follows trajectories with several changes in heading, speed and altitude. The radar tracks are modelled with different types of segments, straight lines, cubic spline function and shape preserving cubic function. The longitudinal, lateral and vertical deviations are calculated for terminal manoeuvring area scenarios. The most promising model of the radar tracks resulted from a mixed interpolation using straight lines for linear segments and spline cubic functions for curved segments. A sensitivity analysis is used to optimise the size of the window for the segmentation process.
\end{abstract}

\section{Keywords}

Segmentation model, radar data, spline function, software tool, collision risk model

\section{Introduction}

The air transport is a strategic sector for the economic growth of any country. To keep the stability and development of this transport mode it is necessary to establish a safe and efficient operational system with a solid safety management system (SMS).

The SMS in the aviation sector is responsible for ensuring an acceptable level of the risk of damage for the people and goods through a continuous process of hazard identification and risk management.

The national and international authorities establish the acceptability target level of safety (TLS) as the safety objectives for some operational scenario. Some examples of TLS are defined in different International Civil Aviation Organization (ICAO) working groups, for instance the All-Weather Operational Panel (AWOP) established the risk of accident in approach or landing phase due to any possible cause as $1.5 \times 10^{-7}$ per flight. $^{1}$

The safety evaluation procedure has to be performed periodically to determine if any change in the operation or in the systems is deteriorating the level of safety. Due to the difficulty to evaluate the collision risk, a set of indicators are used to determine indirectly the safety performance in an operational scenario. Two examples of these indicators are the number of losses of separation between two aircrafts in an en route airspace or the number of runway incursions in an air traffic control tower. ${ }^{2}$

Nevertheless, the method to assess safety based on these indicators has two weaknesses: it depends on the willingness of pilots and air traffic controllers (ATC) to notify air traffic incidents, which is not uniform for all countries, ${ }^{3}$ but even more important, it does not provide a value of collision risk for an operational scenario.

Over the last 40 years different models have been developed to estimate the collision risk between aircraft. In the early $1960 \mathrm{~s}, \mathrm{Marks}^{4}$ defined the principles of the collision risk model (CRM) and Reich ${ }^{5}$ developed the initial CRM in 1966. The Reich model estimates the collision risk between two en route level flying aircraft. The main objective of this model is the determination of lateral separation between adjacent flight levels; ICAO used it in the North Atlantic Organised Track System. ${ }^{6}$

In the past years new CRMs were developed to improve some of the limitations of the Reich model. Bakker and Blom ${ }^{7}$ refined the model weakening the assumption about independence between position and speed of aircraft. Brooker ${ }^{8}$ extended it to an en route controlled airspace. Carpenter and Kuchar, ${ }^{9}$ Powell and Sharon, ${ }^{10}$ Kuchar and Winder, ${ }^{11}$ Winder and Kuchar ${ }^{12}$ and Powell ${ }^{13}$ developed models for landing

\footnotetext{
'Aena, Madrid, Spain

${ }^{2}$ Polytechnic University of Madrid, Madrid, Spain

Corresponding author:

JA Cózar, Aena, Crta de la Base Km. 0,800 28850 Torrejón de Ardoz, Madrid, Spain.

Email: jacozar@aena.es
} 
phase on closely and ultra closely spaced runways or Shepherd et al. ${ }^{14}$ for final approach.

However, none of the previous models is appropriate to assess and monitor the level of safety in high density en route or terminal manoeuvring area (TMA) radar airspaces like in Europe, with high amount of traffic, a large number of crossings tracks, climbing and descending aircrafts and complicated route structure.

The main limitations of these CRMs are: ${ }^{15}$

- Deviation from nominal rotute. Classical models are based on the estimation of position deviation with respect to the nominal route followed by each aircraft. This assumption is valid in an oceanic airspace or approaching to an airport, but not in a continental airspace with some aircraft following heading instruction from air traffic control.

- Human factor. This issue is not usually included in classical CRMs, but ATC is an important contributor to mitigate the risk.

- Restlt validation. The difficulty to obtain reliable statistical data to validate the models from rare events such as mid-air collision derives in conservative assumptions not useful in complex scenarios.

- Complex theoretic formulation. The extension of the classical models to complex scenarios requires sophisticated mathematical formulations that imply a difficult understanding and an important computational effort.

- Limitations of ctorrent stochastic models. The current stochastic models do not allow to model the effect, on the risk of collision, of a given procedure, or of a device such as a short-term conflict alert.

- Risk exposure in current airspace. Many CRMs deal with obtaining passing frequencies for aircraft on adjacent flight levels, but in complex scenarios the analysis of radar data needs to incorporate all possible events such as aircraft overtaking, aircraft crossing on the same flight level and aircraft climbing/descending in the vicinity of other traffic.

As a reaction to overcome these limitations, there is a new line of research for a three-dimensional (3D) CRM that uses a significant source of information such as the aircraft radar tracks. ${ }^{16}$ This 3D CRM assesses the level of safety in en route airspace, where ATC monitors air traffic by means of radar and provides tactical instructions to aircraft. This model is based on the mathematical formulation developed by Burt ${ }^{17}$ that estimates the average probability of potential collision distinguishing four different aircraft encounter geometries.

The rationale behind the $3 \mathrm{D}$ CRM is that aircraft collisions are exceptional events, then the probability of occurrence cannot be inferred by direct observation. These models estimate the risks of an airspace based on more frequent events that could lead to an air collision, like the losses of separation among airplanes.
The availability of flight radar data could also help to test different analytical models of human behaviour (either ATC or aircrew) by identifying the traces of interventions in the radar track and the associated reaction times.

The development of a 3D CRM for a high density terminal area, where the operational concept is more complex than in an en route scenario, needs both an appropriate management of the aircraft radar tracks to model the aircraft trajectories to reduce the computational effort without loss of relevant information by optimum interpolation minimising the errors between the real and the interpolated tracks.

\section{Concept of operation in a terminal area}

The operation in a TMA consists basically in feeding air traffic to the internal airfields and allowing departing traffic to be integrated efficiently and safely in route airspace. Aircraft usually follow standardised departure and arrival routes, allowing ordered sequence, but nevertheless, these routes can be modified by instructions from air traffic control for safety or efficiency reasons.

Traffic flow in a TMA is a combination of departing and arriving traffic that the route structure and the operational design attempts to segregate minimising crossing points. When following standardised routes, the actual tracks exhibit lateral and vertical deviations from the nominal route depending upon the navigation performance used by the aircraft.

The flight routes in a TMA present more dispersion than in an en route scenario. ${ }^{18}$ Madrid Barajas airport has four runways being the preferential configuration $32 \mathrm{R}$ and $32 \mathrm{~L}$ runways for arrivals and $36 \mathrm{R}$ and $36 \mathrm{~L}$ runways for departures. A sample day of flights in the Madrid TMA (1284 flights) is analysed to show the main differences with en route traffic.

Figure 1 shows the lateral and vertical dispersion from nominal route for flights arriving from the Northeast to the $32 \mathrm{R}$ runway of Madrid Barajas airport. The dispersion in the trajectories $(40 \mathrm{~km}$ of lateral dispersion and $3 \mathrm{~km}$ of vertical dispersion) suggests that the method to model them, requiring retention of the maximum amount of detail, has to be more complex than the segmentation process used for en route traffic.

Similarly, heading, ground speed and rate of climb or descent (ROCD) of flights in that flow arriving to Madrid Barajas airport also presented a relevant dispersion, more than 200 knot in speed, about $1500 \mathrm{fpm}$ in rate of descent and $50^{\circ}$ in heading (see Figure 2).

Table 1 contains the percentage of time that flights are turning versus maintaining their direction, as well as the time they are climbing and descending compared to level flight for different traffic samples in Madrid TMA.

This information reveals that more than $20 \%$ of the time aircrafts are turning and less than $20 \%$ of 


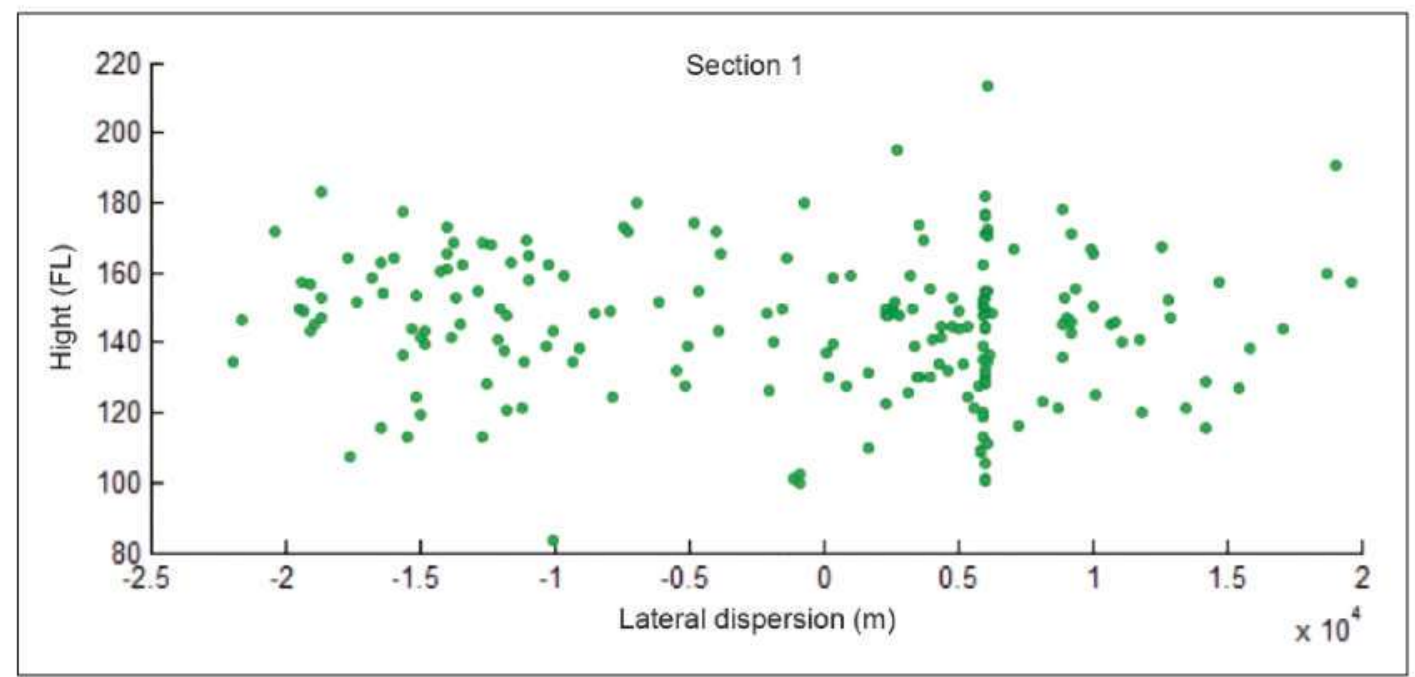

Figure I. Lateral and vertical dispersion in traffic arriving from the Northeast to $32 \mathrm{R}$ runway to Madrid-Barajas airport.

the time they are in level flight. Therefore, radar tracks could be modelled using a combination of straight line segments, that is approaching the turns by an adequate number of straight sections ${ }^{19}$ or using a combination of straights and curves depending on the type of segments. But, according to previous data, it can be foreseen that there will be a better fit to the real track when the segmentation process is based on curves or a combination of curves and straight lines.

Moreover, to have similar deviations from actual track by using straight segments, a large number of these will be required, demanding a higher computational effort than if using a mixed segmentation where the turns are modelled with curves.

\section{Radar track segmentation with straight and curved segments}

One of the goals of segmentation is to replace the huge amount of points of the radar track representing aircraft trajectory by a minimum sequence of straight or curved segments. ${ }^{20}$ The resulting segmented track is then characterised by a ordered sequence of points defined by their coordinates, time, speed, heading and climbing rate for each segment. Furthermore, arcs will yield the parameters that define the curve.

In order to detect the behaviour of the aircraft, the segmentation process starts obtaining statistical values of the radar track. To this end the mean and standard deviation (STD) of the heading, and speed and rate of climb/descent are calculated as follows.

- Mean estimator

$$
\bar{x}=\frac{1}{n} \sum x_{i}
$$

Confidence interval for the mean estimator with unknown variance

$$
\left(\bar{x}-z_{\frac{\alpha}{2}} \frac{s}{\sqrt{n}}, \bar{x}+z_{\frac{\alpha}{2}} \frac{s}{\sqrt{n}}\right)
$$

$n$ : number of samples used to estimate

$s:$ standard deviation estimator

$z_{q}: q$ th centile of $N(0,1)$

$(1-\alpha)$ : confidence level

- Standard deviation estimator

$$
s=\sqrt{\frac{\sum(x i-\bar{x})^{2}}{n}}
$$

It can be demonstrated that $\mathrm{s}$ follows a Chisquared distribution $\left(\chi_{n}^{2}\right)$ with $n-1$ degree of freedom as in equation (4)

$$
\frac{n s^{2}}{\sigma^{2}}=\chi_{n-1}^{2}
$$

The confidence interval of $\sigma$ for the estimator s with a given level of confidence $(\alpha)$

1. Interval of confidence of $\sigma$ with two-tailed distribution

$$
\left(s \sqrt{\frac{n \cdot}{\chi_{\frac{2}{2}}^{2}}}, s \sqrt{\frac{n \cdot}{\chi_{1-\frac{\omega}{2}}^{2}}}\right)
$$

2. Interval of confidence of $\sigma$ with one-tailed distribution

$$
\left(0, s \sqrt{\frac{n \cdot}{\chi_{1-\alpha}^{2}}}\right)
$$




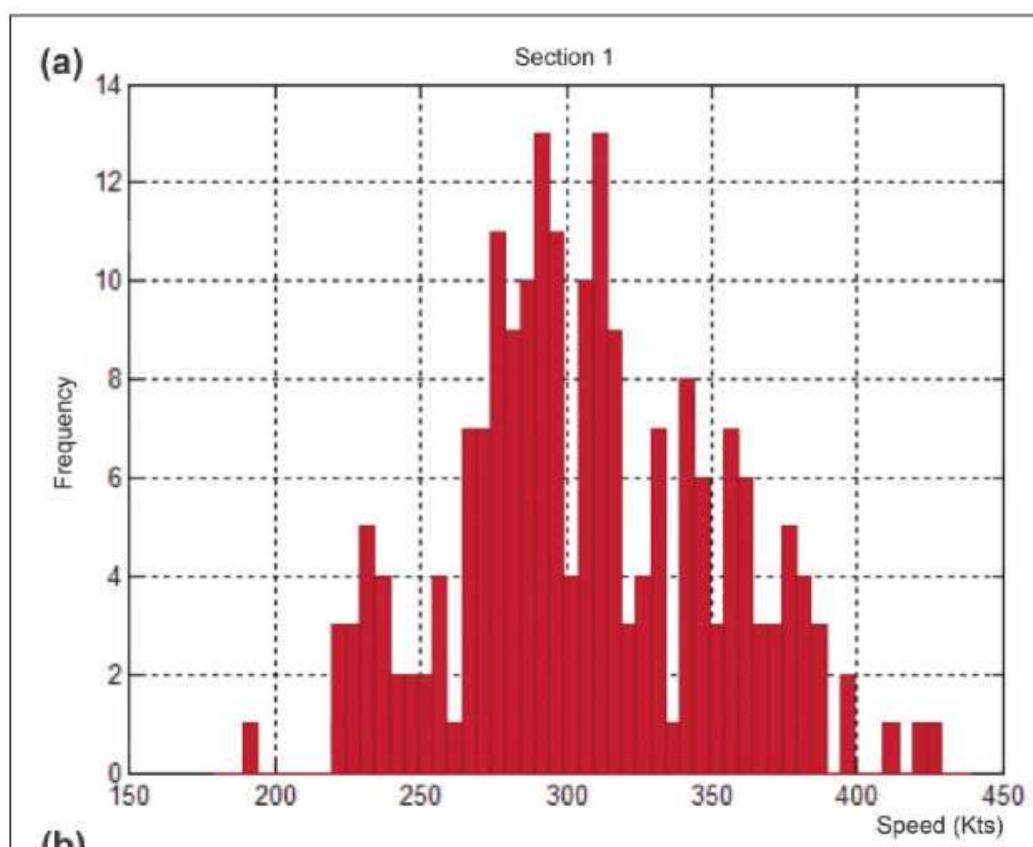

(b)
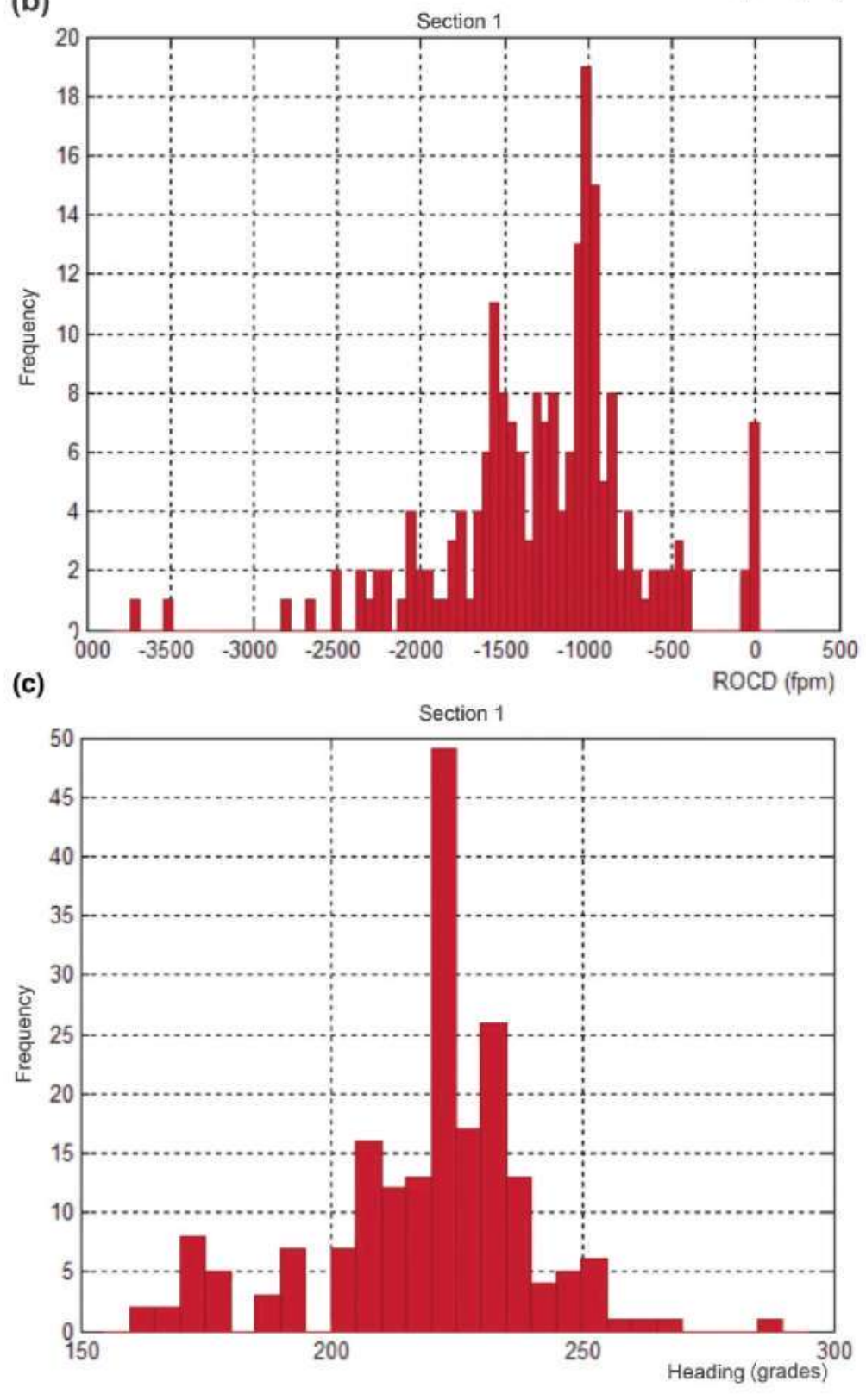

Figure 2. Dispersion values for (a) ground speed dispersion, (b) rate of climb/descent dispersion, (c) heading dispersion in traffic arriving from the Northeast to $32 \mathrm{R}$ runway to Madrid-Barajas airport. 
Table I. Analysis of tracks in Madrid terminal area.

\begin{tabular}{|c|c|c|c|c|c|c|c|c|c|}
\hline$N^{\circ}$ flights & $\begin{array}{l}\text { Total time } \\
\text { flown (min) }\end{array}$ & $\begin{array}{l}\text { Flight time } \\
\text { turning (min) }\end{array}$ & $\%$ & $\begin{array}{l}\text { Flight time } \\
\text { level (min) }\end{array}$ & $\%$ & $\begin{array}{l}\text { Flight time } \\
\text { descending ( } \mathrm{min})\end{array}$ & $\%$ & $\begin{array}{l}\text { Flight time } \\
\text { ascending ( } \min )\end{array}$ & $\%$ \\
\hline 1201 & $26,050.0$ & 5643.8 & 22 & 4876.5 & 19 & $12,05 \mid .3$ & 46 & 9122.3 & 35 \\
\hline 1284 & $25,481.4$ & 5787.5 & 23 & 3207.0 & 13 & $12,540.2$ & 49 & 9734.2 & 38 \\
\hline 1504 & $30,047.9$ & 6672.7 & 22 & $56 \mid 4.3$ & 19 & $14,259.3$ & 47 & $10,174.3$ & 34 \\
\hline 1078 & $22,578.7$ & 4430.1 & 20 & 3449.9 & 15 & $10,906.3$ & 48 & 8222.5 & 36 \\
\hline
\end{tabular}

These values are calculated along the track with samples of a given number of points. The sample size or window cannot be exceptionally large because changes in path or speed of the aircraft in short time would not be detected and not too small because it would imply an excessive number of segmentation points and even errors in the database of the radar tracks. $^{21}$

The track statistics are compared with reference values obtained from empirical assessment of the performance of a significant number of flights, which allows the determination of the moment when the flight is turning, changing its speed or its flight level.

The change of heading points is established when the standard deviation of the samples from the track reaches the value 3.0. To detect paired turns, a second limit value 2.0 in the standard deviation is also proposed, and thus, when two turns are close, although the latter is of a minor deviation, both come together like just one single turn, which avoids excessive segmentation points.

Thereby segmentation points are established at the beginning and ending turns. Furthermore, when turns are over $70^{\circ}$ two additional segmentation points are also included to best fit the intermediate segmented track.

Level flight segments are assumed when averaged the rate of climb/descent speed is inside the upper and lower limits 310 and $-300 \mathrm{ft} / \mathrm{min}$. Similarly segmentation points are situated in the ascending and descending segments when standard deviation presents local maxima.

For ground speed modelling is a little different because it is difficult to find constant speed sections, so segmentation points are established when standard deviation is above the value 3 .

Figure 3 shows an example of the segmentation points following the limits described earlier.

Once determined the ends of the segments marking turns, segments of ascent or descent and significant changes in speed, from time, position and speed a straight segmented track can be generated with equation $(7)^{20}$

$$
R_{i}=\left\{\begin{array}{l}
{\left[t_{i 0}, x_{i 0}, y_{i 0}, h_{i 0}, v_{i 0}, \dot{h_{i 0}}\right],\left[t_{i 1}, x_{i 1}, y_{i 1}, h_{i 1}, v_{i 1}, \dot{h}_{i 1}\right], \ldots} \\
,\left[t_{i n}, x_{i n}, y_{i n}, h_{i n}, v_{i n}, \dot{h_{i n}}\right]
\end{array}\right\}
$$

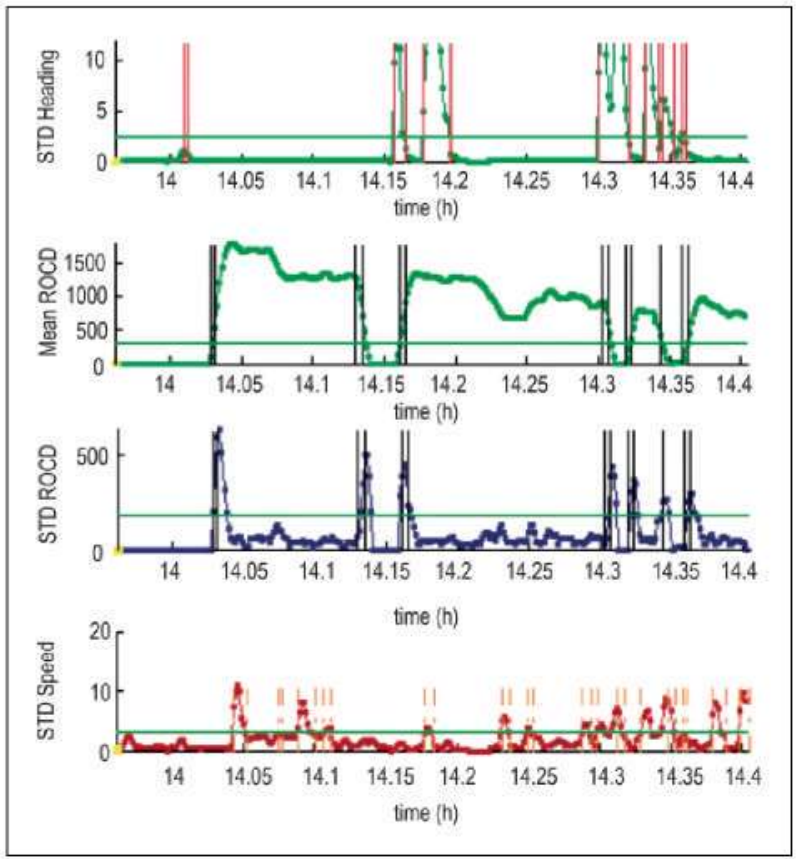

Figure 3. Segmentation points example based on heading, ROCD and ground speed.

For curved segmentation, two types of interpolation are used: cubic spline function and the 'shape preserving' cubic spline function. ${ }^{22}$

1. Cubic spline function. The interpolation with the spline function, also called segmental polynomial interpolation is based on the use of polynomial segments and an adequate merge of these to ensure continuity conditions.

Given a set of $n+1$ points $\left\{x_{i}, y_{i}\right\}$ a cubic spline function on these points is a function $\mathrm{S}$ which verifies

$$
\begin{aligned}
& S(x)=q_{k}(x) \text { polinomial of degree } \leqslant 3, x \in\left[x_{k}, x_{k+1}\right] \text {, } \\
& \text { (i) } S\left(x_{k}\right)=y_{k}, k=0,1, \ldots, n \\
& \text { (ii) } S_{k+1}\left(x_{k}\right)=S_{k}\left(x_{k}\right), k=1, \ldots, n \\
& \text { (iii) } S_{k+1}^{\prime}\left(x_{k}\right)=S_{k}^{\prime}\left(x_{k}\right) \text { and } S_{k+1}^{\prime \prime}\left(x_{k}\right)=S_{k}^{\prime \prime}\left(x_{k}\right), \\
& k=1, \ldots, n \\
& \text { (iv) } S^{\prime \prime}\left(x_{0}\right)=S^{\prime \prime}\left(x_{n}\right)=0
\end{aligned}
$$

Using the spline interpolation to a curved segment of $n+1$ points obtained from a radar track segmented 


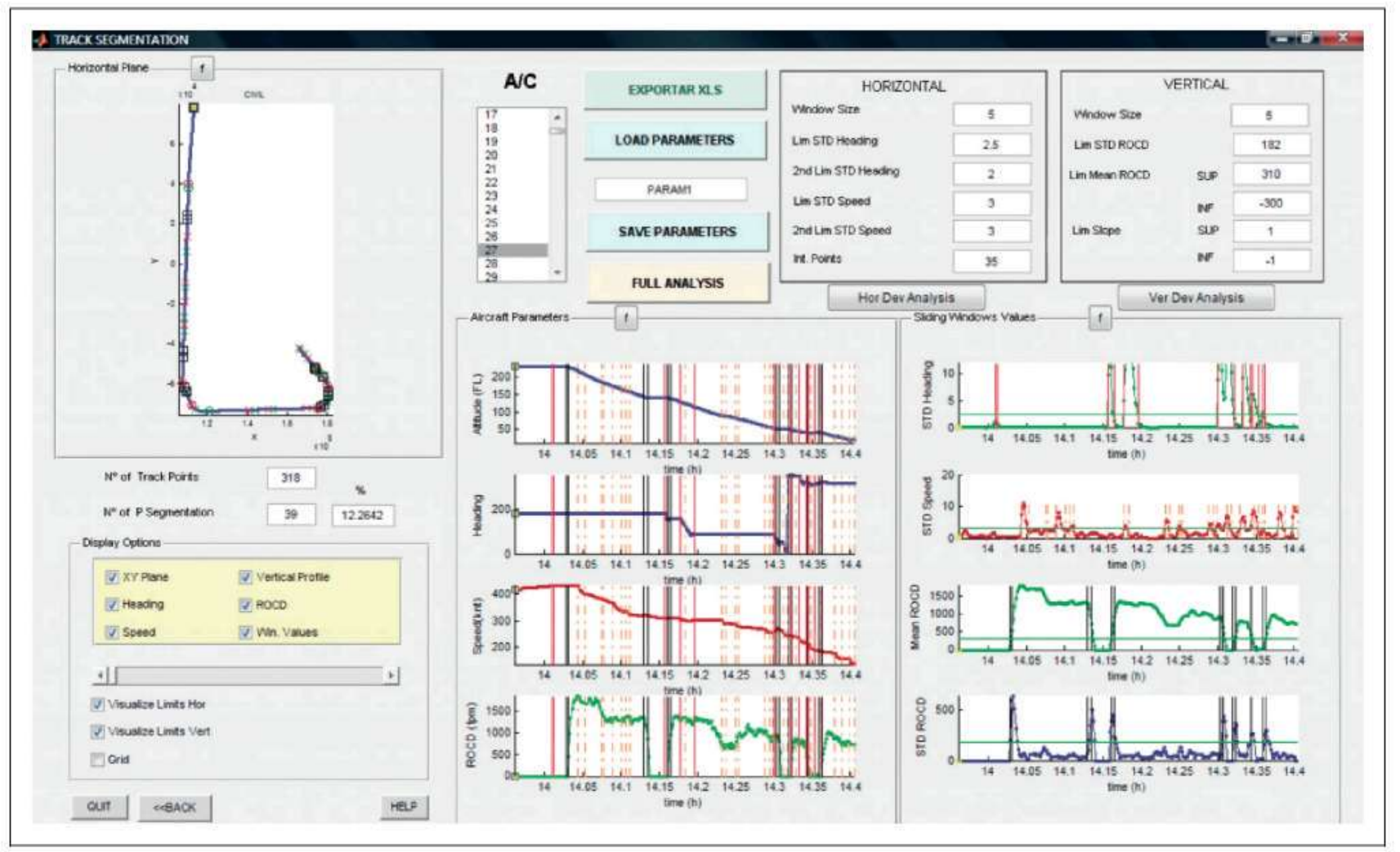

Figure 4. Segmentation tool interface.

such that $\left(t_{k}, x_{k}, y_{k}, h_{k}\right)$ for $k=0, \ldots, n$, the cubic spline function $S(t)$ on that segment would be

$S(t)=\left(X_{k}(t), Y_{k}(t), H_{k}(t)\right)$ being $X$,

$Y$ y $H$ polinomial of degree $\leqslant 3, t \in\left[t_{k}, t_{k+1}\right]$,

(i) $S\left(t_{k}\right)=\left(x_{k}, y_{k}, h_{k}\right), k=0,1, \ldots, n$

(ii) $S_{k+1}\left(t_{k}\right)=S_{k}\left(t_{k}\right), k=1, \ldots, n$

(iii) $S_{k+1}^{\prime}\left(t_{k}\right)=S_{k}^{\prime}\left(t_{k}\right)$ and $S_{k+1}^{\prime \prime}\left(t_{k}\right)=S_{k}^{\prime \prime}\left(t_{k}\right)$,

$k=1, \ldots, n$

(iv) $S^{\prime \prime}\left(t_{0}\right)=S^{\prime \prime}\left(t_{n}\right)=0$

2. 'Shape preserving' cubic spline function. The second method of interpolation for curved segments is called 'shape preserving' cubic spline, determining the first derivatives of the interpolation function which does not cause excessive peaks.

Given a group of $n+1$ points $\left\{x_{i}, y_{i}\right\}, \delta_{k}$ is defined as the slope of the line joining the two ends of a segment $k$

$$
\delta_{k}=\frac{y_{k+1}-y_{k}}{h_{k}}
$$

Being $h_{k}=x_{k+1}-x_{k}$ the length of sub-interval $k$ and $y_{k}$ the value taken by the function at the end $k$.
A 'shape preserving' cubic spline function on these points is a function $S$ which verifies

$$
\begin{aligned}
& S(x)=q_{k}(x) \text { polinomial of degree } \leqslant 3, x \in\left[x_{k}, x_{k+1}\right] \text {, } \\
& \text { (i) } S\left(x_{k}\right)=y_{k}, k=0,1, \ldots, n \\
& \text { (ii) } S_{k+1}\left(x_{k}\right)=S_{k}\left(x_{k}\right), k=1, \ldots, n
\end{aligned}
$$

In addition, the derivatives at the ends points are determined with the following rules. If $\delta_{k}$ and $\delta_{k+1}$ have opposite signs, or if both are zero, then $x_{k}$ is a local minimum or maximum and, therefore, the derivative is zero, $S_{k}^{\prime}\left(x_{k}\right)=S_{k+1}^{\prime}\left(x_{k}\right)=0$, but unlike the first method discontinuity occurs in the second derivative.

If $\delta_{k}$ and $\delta_{k+1}$ have the same sign but different values and the intervals are the length, $h_{k}$, the expression for calculating the derivative is

$$
\frac{1}{S_{k}^{\prime}\left(x_{k}\right)}=\frac{1}{S_{k+1}^{\prime}\left(x_{k}\right)}=\frac{1}{2}\left(\frac{1}{\delta_{k+1}}+\frac{1}{\delta_{k}}\right)
$$

As a result, the slope of the polynomial interpolator in this method is the average of the two slopes of the linear interpolation segmental. Interpolation in this case also has two different cubic segments which share the same value of the derivative at the centre and also a discontinuity occurs in the second derivative. 
In the case of $\delta_{k}$ and $\delta_{k+1}$ having the same sign, but the length of the sections being different, the average is a weighted harmonic mean with the length of the segments.

Two additional conditions are needed to determine the derivatives in the first and last point of the curved segment, which are obtained by taking the slope values in the first and last curved segments, respectively

$$
S_{1}^{\prime}\left(x_{0}\right)=\delta_{1}, \quad S_{n}^{\prime}\left(x_{n}\right)=\delta_{n}
$$

The first method described earlier produces oscillations on radar track segmentation that generates significant deviations and then also results from the second approach are presented which produces a smooth interpolation.

\section{Software tool to perform segmentation}

With the purpose of testing the performance of the three types of segmentation the tool developed in Sáez et al. ${ }^{16}$ has been updated in MATLAB ${ }^{0}$ to deal efficiently with large volume files of flight radar tracks.

The tool interface allows the modification of the different parameters used for segmentation: the size of the window (number of points); the standard deviation criteria for heading, speed and rate of climb/ descent; and averaged rate of climb/descent and gradient (see Figure 4).

The tool works flight to flight, presenting results as graphs which represent the evolution of the various statistical and segmentation points detected by the software from the established limits. This allows the analysis of the process of segmentation and to identify improvements.

The massive computing module can automatically segment a complete file of radar tracks and calculate statistical errors produced by segmentation on the actual radar track.

\section{Comparison of results using different types of segmentation}

The results when processing a large number of tracks, using the different methods exposed previously, are here analysed in order to determine which one offers the best performance and smaller deviations from the actual tracks and, therefore, would be a better approximation modelling segmented trajectories within TMA scenarios.

The segmentation tool has been developed to analyse the deviations of individual tracks or massive numbers of tracks with the following options:

- All linear segments, for straight lines and curves

- Linear segments for straight lines and cubic spline functions for curves and
- 'Shape preserving' cubic spline functions for straight and curved segments.

Linear segmentation should a priori give the poorest adjustment when the track has turns, otherwise, the number of required points demanded to maintain small lateral deviations would be very high. With a mixed segmentation errors in turns should be reduced, to this purpose, the cubic spline was used. Oscillation errors mentioned before are not relevant when the segmentation process has a significant number of straight segments.

Additionally, results from a curved segmentation, in this case using 'shape preserving' cubic spline functions - called in MATLAB ${ }^{\circ}$ pchip function - are also included, because oscillation errors occur using cubic spline functions to interconnect a large number of curved segments or arcs.

The longitudinal and lateral deviations calculated for the three models using a sample track are presented in Figures 5 to 7.

Similarly in Figures 8 to 10 the vertical deviations for the three models using the same sample track are presented.

Table 2 compares the deviations for the three segmentation process based on the main error indicators represented in Figures 5 to 10.

The results for the sample radar track indicate that mixed segmentation with straight lines and cubic spline functions is significantly better than the other two models, generating lower lateral and longitudinal errors. The mean absolute lateral deviation is $25.7 \mathrm{~m}$ for the mixed segmentation, while it is 52.2 and $68.5 \mathrm{~m}$ for the linear and curve segmentation, respectively. For the longitudinal deviation the differences are not as relevant, the mean absolute longitudinal deviations are $38.2 \mathrm{~m}$ with mixed segmentation, $40.7 \mathrm{~m}$ with linear segmentation and $63.9 \mathrm{~m}$ with curved segmentation.

The comparison for the sample track shows similar values in the mean absolute vertical error for the linear and mixed segmentation 33.5 and $34.7 \mathrm{ft}$, respectively, which are lower than for the curved segmentation $80.8 \mathrm{ft}$.

Using the test for more relevant data samples of 4 days of traffic in Madrid TMA, the results obtained are exposed in Tables 3 to 6 .

Tables 3 to 5 shows that deviations depend on the level of traffic, a higher number of aircraft means that the air traffic control has to deviate the aircrafts from the standard route of arrival with vectors or use the holding points to sequence the traffic, then the lateral and longitudinal deviations are higher due to longer flights with more turns. In addition, it can be observed in Table 6 that the Madrid TMA operation with the non-preferential configuration (south configuration) is more complex, which is the rationale behind the higher deviations in the Day 4 analysis. 


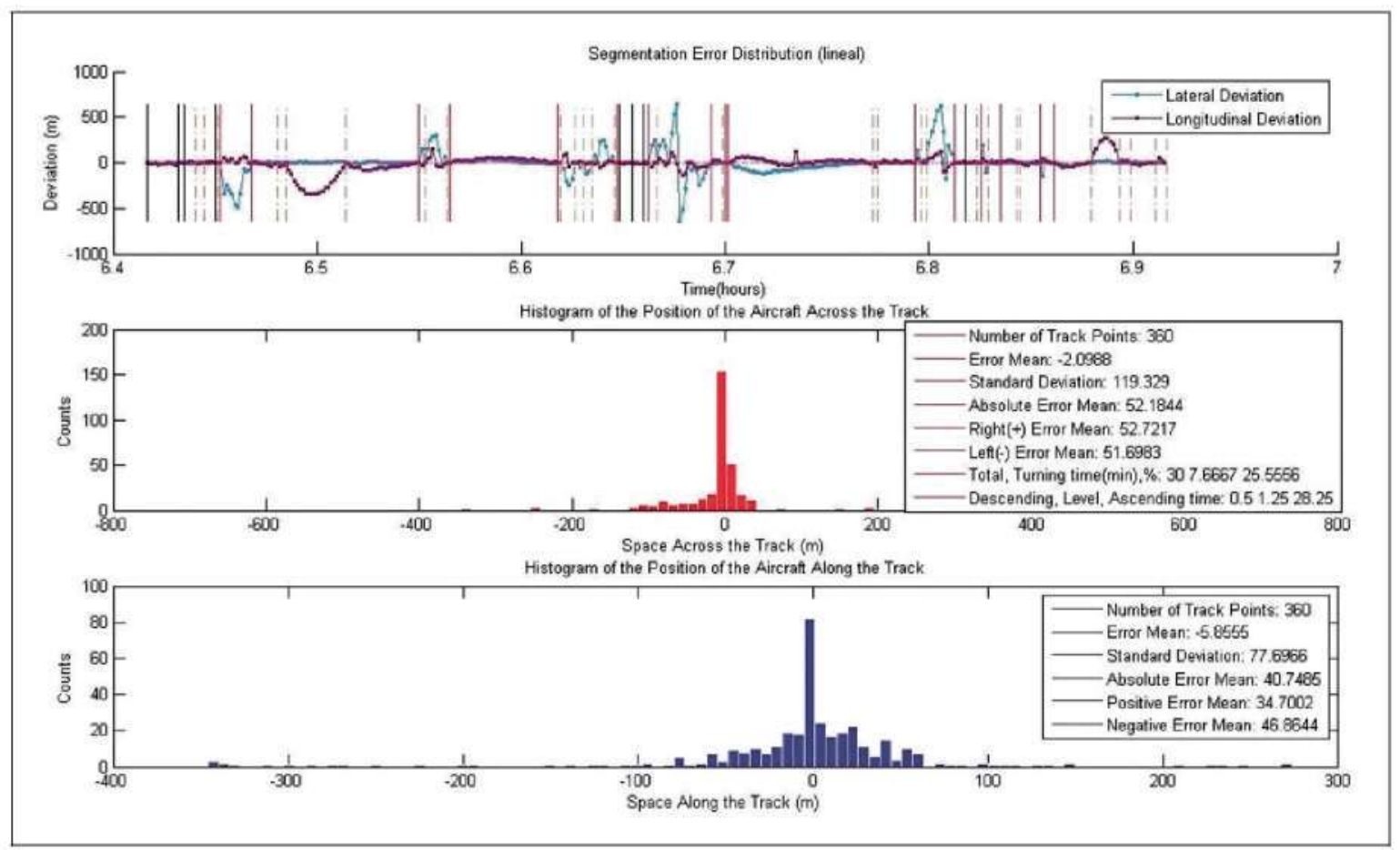

Figure 5. Lateral and longitudinal deviations for a sample track using linear segmentation.

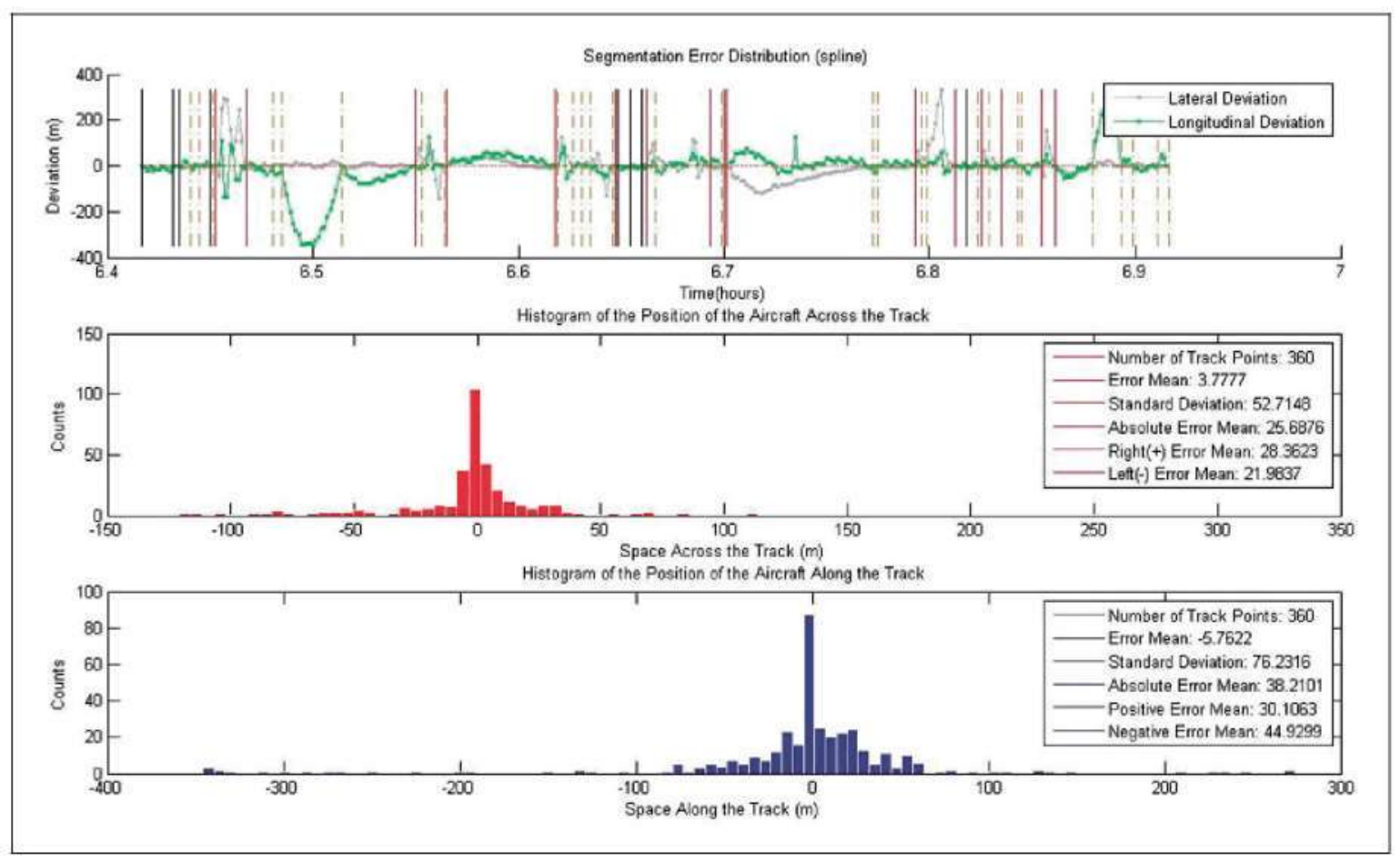

Figure 6. Lateral and longitudinal deviations for a sample track using mixed segmentation (spline).

The results confirm (see Tables 3 to 7 ) the initial conclusions drawn from the first analysis where a sample track was considered and then the conclusion is that the mixed segmentation (linear-spline function) is the best model for horizontal track segmentation. Table 7 for the 4 days traffic sample presents a lateral deviation of $33.9 \mathrm{~m}$ and a longitudinal deviation of $67.5 \mathrm{~m}$.

The linear and mixed segmentations exhibit similar results for the vertical profile. Table 7 for the 4 days traffic sample shows a vertical deviation of 46.6 and $46.0 \mathrm{ft}$ for linear and mixed segmentation, 


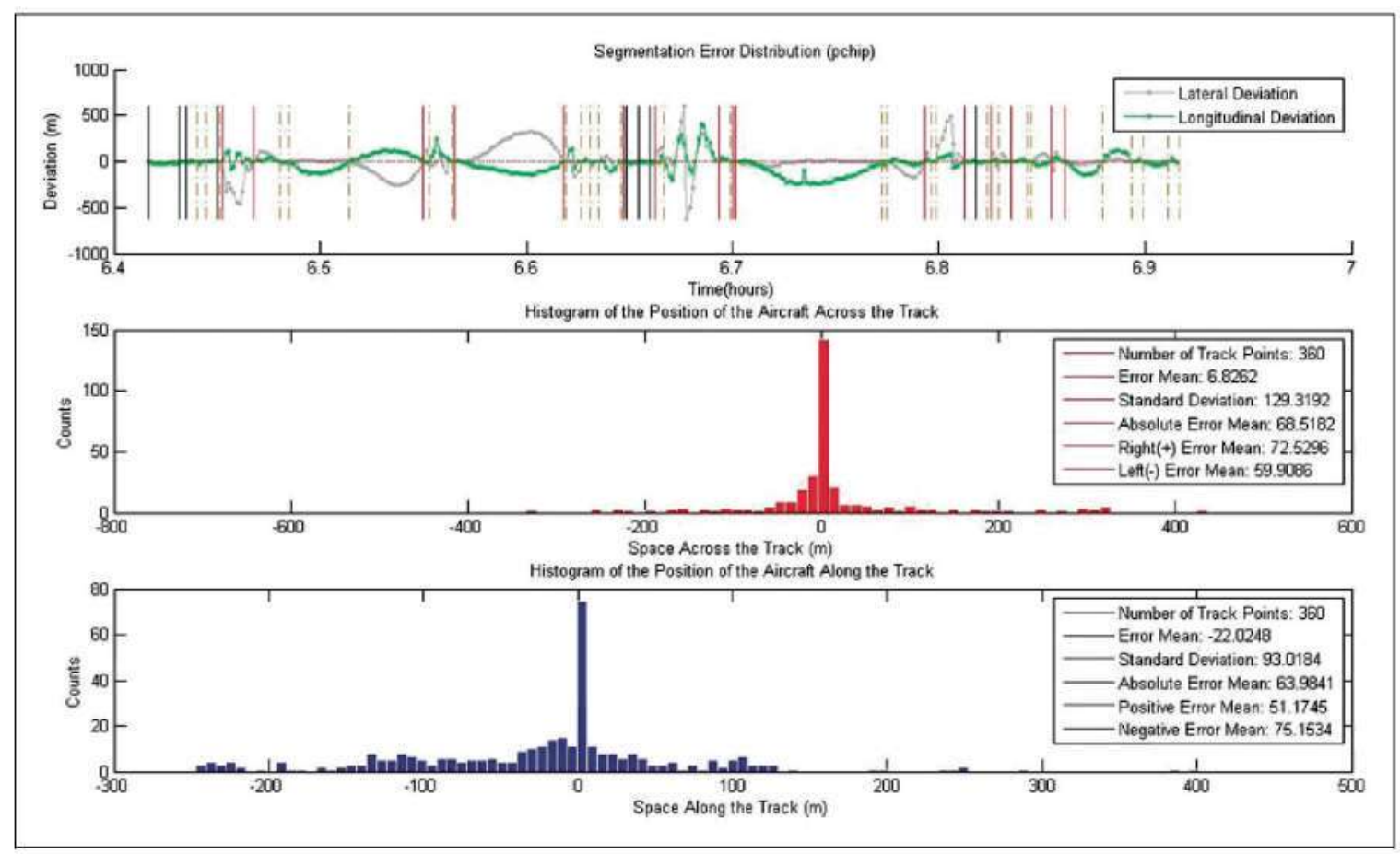

Figure 7. Lateral and longitudinal deviations for a sample track using curved segmentation (pchip).

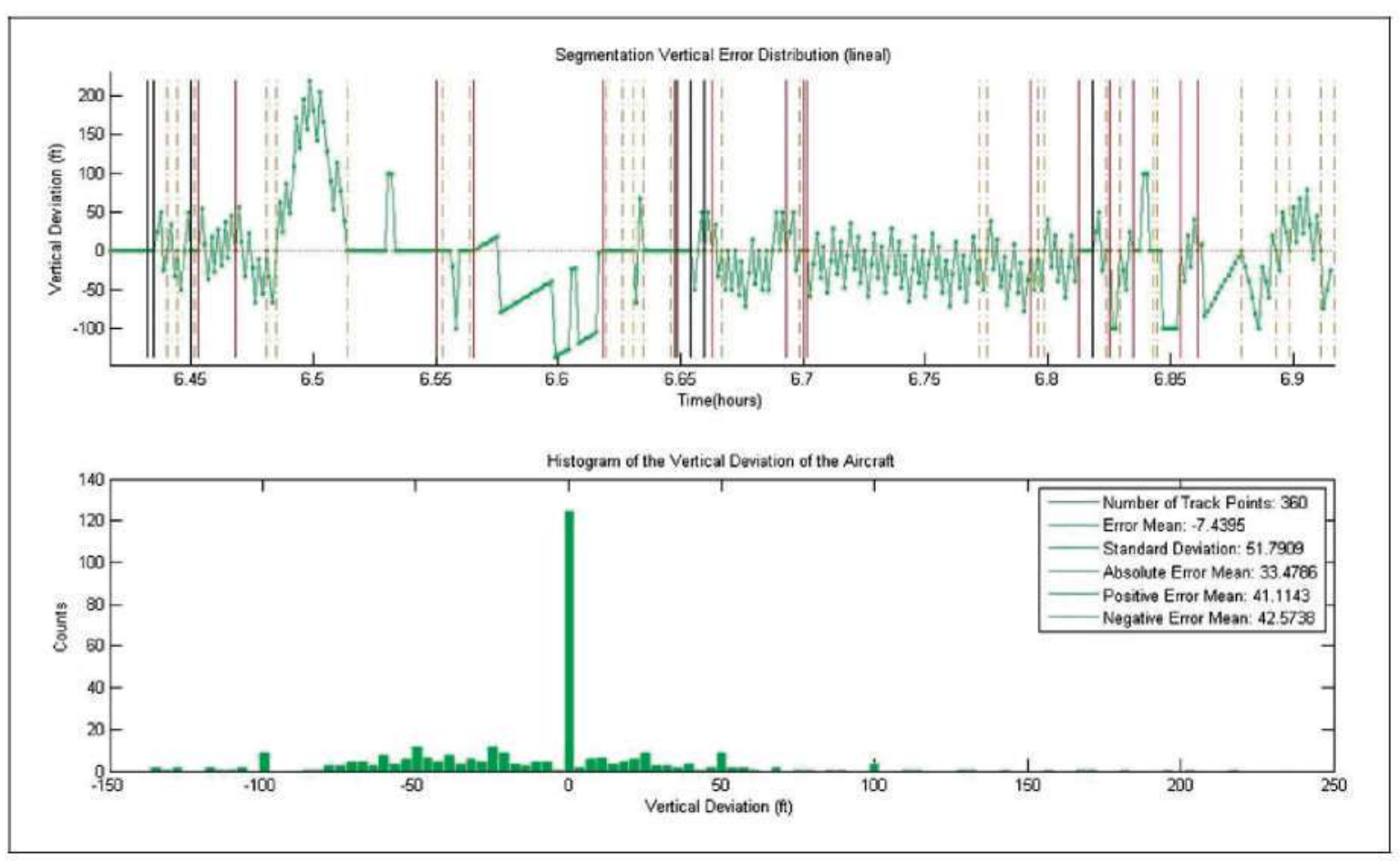

Figure 8. Vertical deviations for a sample track using linear segmentation.

respectively. These data suggest that the ascent/descent profile of the airplanes is close to linear, so they can be modelled by straight segments.

In addition to verify that curved segmentation with cubic spline functions should result in a bad adjustment due to the oscillation errors, a column with the statistics for this segmentation has also been included in the Tables 3 to 7 , where it is shown that all deviations for this segmentation are higher in all the scenarios.

\section{Sensitivity analysis of the window size parameter}

The size of the window used to compute statistics impacts on the segmentation process. The wrong 


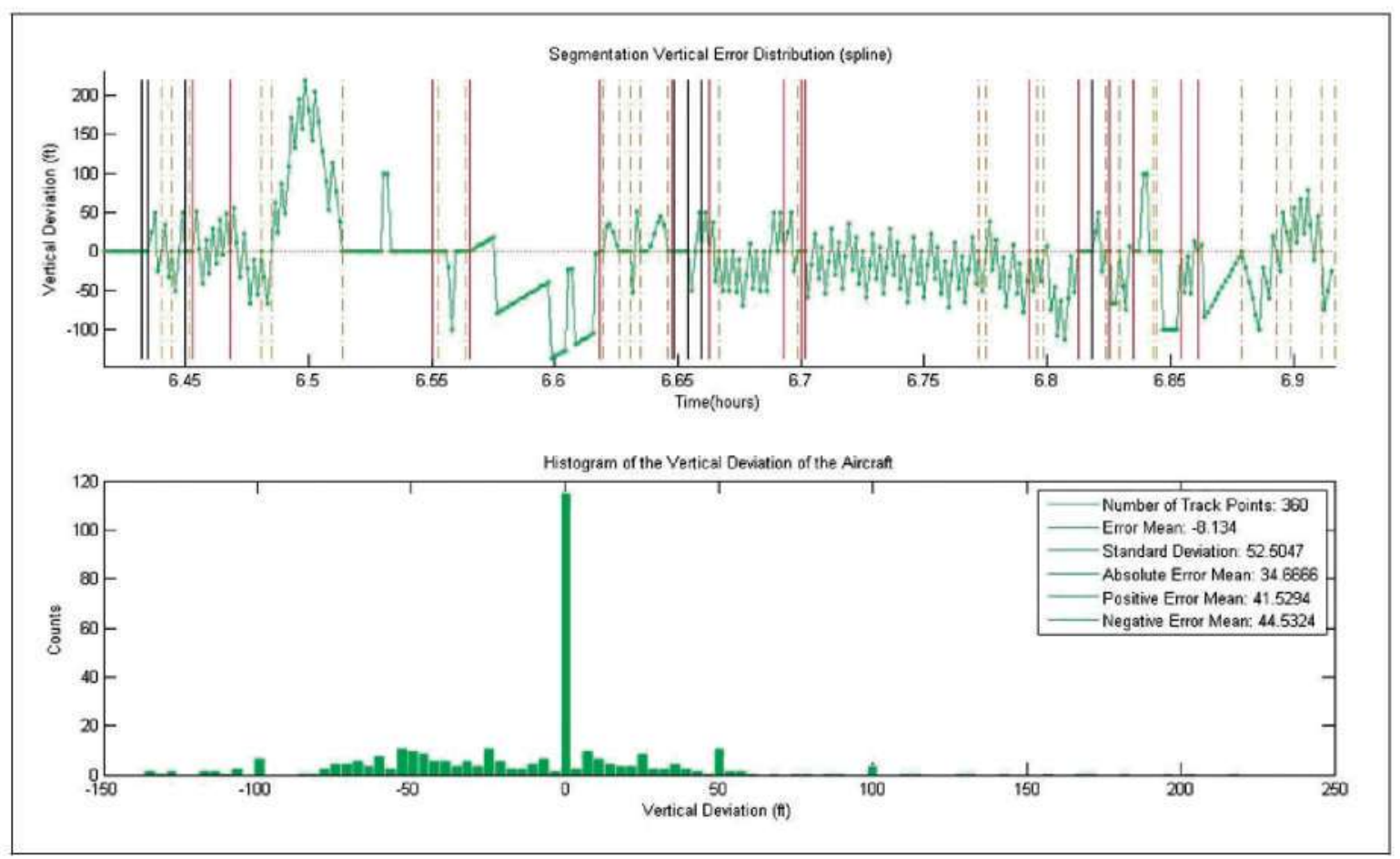

Figure 9. Vertical deviations for a sample track using mixed segmentation (spline).

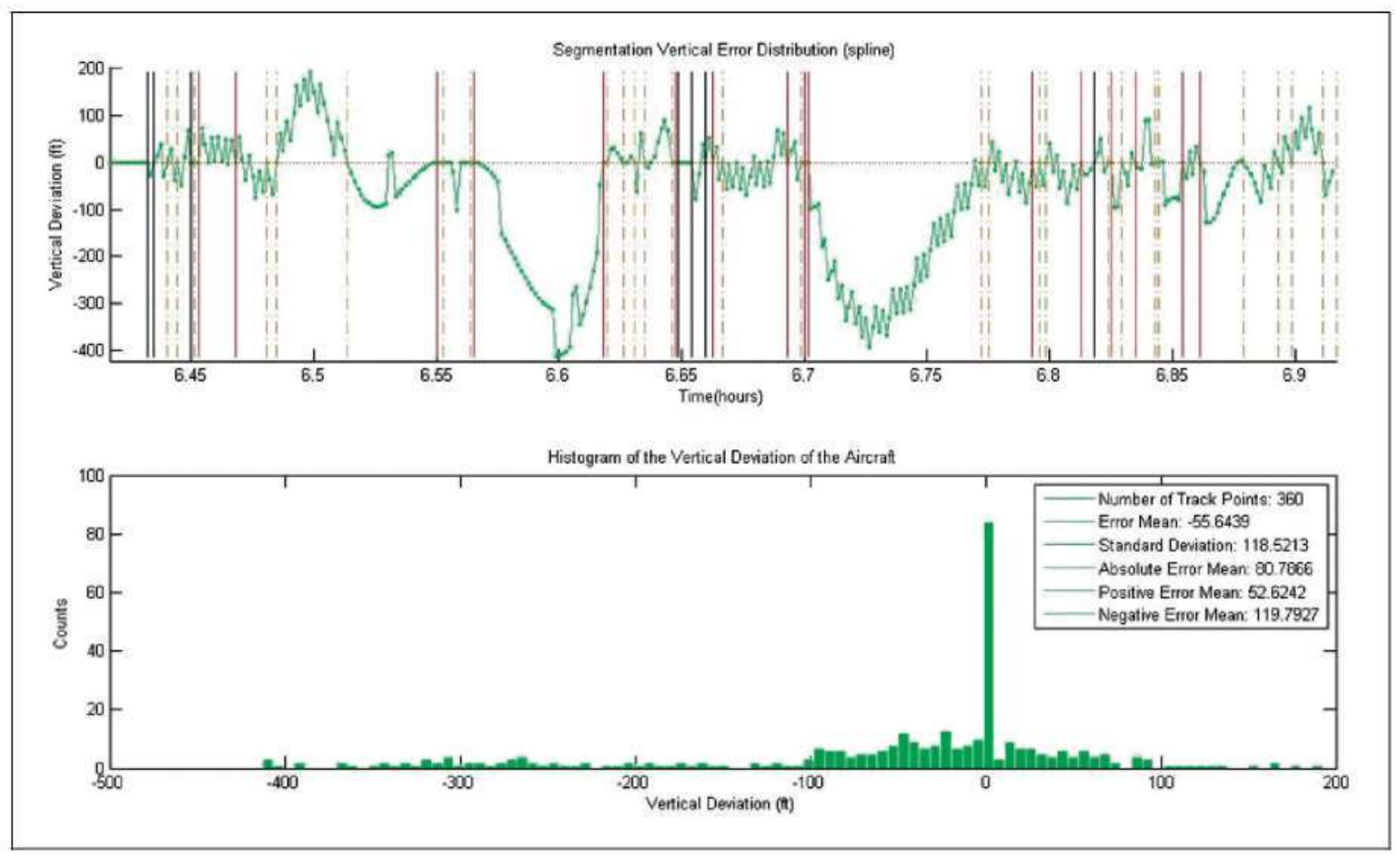

Figure 10. Vertical deviations for a sample track using curved segmentation (pchip).

selection of this parameter generates an incorrect number of segments, either through being excessive or insufficient. Therefore, a sensitivity analysis is required to determine the best option for the window size.

Using a sample of 1552 flights and keeping all other variables fixed, different window sizes are tested. Then, the resulting errors generated with mixed segmentation (linear-spline function) and linear segmentation are presented.

The lateral mean absolute error grows with the window size for both segmentations mixed and linear, but the deviation in the former case is always 
Table 2. Lateral, longitudinal and vertical deviations for the sample track.

\begin{tabular}{lclc}
\hline & Linear & Mixed (spline) & Curved (Pchip) \\
\hline STD lateral deviation $(m)$ & 119.3 & $52.7(-56 \%)$ & $129.3(8 \%)$ \\
Mean absolute lateral deviation $(\mathrm{m})$ & 52.2 & $25.7(-51 \%)$ & $68.5(31 \%)$ \\
STD longitudinal deviation $(\mathrm{m})$ & 77.7 & $76.2(-2 \%)$ & $93.0(20 \%)$ \\
Mean absolute longitudinal deviation $(\mathrm{m})$ & 40.7 & $38.2(-6 \%)$ & $63.9(57 \%)$ \\
STD vertical deviation $(\mathrm{ft})$ & 51.8 & $52.5(1 \%)$ & $118.5(129 \%)$ \\
Mean absolute vertical deviation $(\mathrm{ft})$ & 33.5 & $34.7(3 \%)$ & $80.8(141 \%)$ \\
\hline
\end{tabular}

STD: standard deviation.

Table 3. Lateral, longitudinal and vertical deviations, day I.

\begin{tabular}{lcccc}
\hline 1078 flights & Linear & Mixed (spline) & Curved (pchip) & Curved (spline) \\
\hline Mean absolute lateral deviation (m) & 36.6 & 21.1 & 56.7 & 70.5 \\
Mean STD lateral deviation (m) & 94.6 & 48.2 & 121.6 & 141.6 \\
Mean absolute longitudinal deviation (m) & 61.1 & 59.4 & 56.8 & 61.3 \\
Mean STD longitudinal deviation (m) & 113.1 & 111.1 & 111.2 & 121.0 \\
Mean absolute vertical deviation (ft) & 45.1 & 44.5 & 50.1 & 65.0 \\
Mean STD vertical deviation (ft) & 69.2 & 68.3 & 77.7 & 100.0 \\
\hline
\end{tabular}

STD: standard deviation.

Table 4. Lateral, longitudinal and vertical deviations, day 2.

\begin{tabular}{lcccc}
\hline I504 flights & Linear & Mixed (spline) & Curved (pchip) & Curved (spline) \\
\hline Mean absolute lateral deviation (m) & 49.6 & 32.6 & 66.9 & 100.6 \\
Mean STD lateral deviation $(\mathrm{m})$ & 141.6 & 92.8 & 159.6 & 209.7 \\
Mean absolute longitudinal deviation (m) & 62.9 & 61.8 & 62.1 & 87.1 \\
Mean STD longitudinal deviation (m) & 139.2 & 138.8 & 143.2 & 186.1 \\
Mean absolute vertical deviation (ft) & 47.1 & 46.5 & 52.1 & 67.3 \\
Mean STD vertical deviation (ft) & 78.0 & 77.1 & 86.3 & 109.2 \\
\hline
\end{tabular}

STD; standard deviation.

Table 5. Lateral, longitudinal and vertical deviations, day 3.

\begin{tabular}{lcccc}
\hline I284 flights & Linear & Mixed (spline) & Curved (pchip) & Curved (spline) \\
\hline Mean absolute lateral deviation $(\mathrm{m})$ & 42.2 & 24.3 & 56.7 & 67.9 \\
Mean STD lateral deviation $(\mathrm{m})$ & 113.3 & 61.8 & 127.3 & 140.9 \\
Mean absolute longitudinal deviation $(\mathrm{m})$ & 51.2 & 49.1 & 42.3 & 42.6 \\
Mean STD longitudinal deviation (m) & 92.6 & 91.5 & 81.1 & 80.4 \\
Mean absolute vertical deviation (ft) & 46.0 & 45.2 & 51.3 & 63.0 \\
Mean STD vertical deviation (ft) & 71.8 & 71.1 & 80.0 & 97.8
\end{tabular}

STD: standard deviation.

Table 6. Lateral, longitudinal and vertical deviations, day 4.

\begin{tabular}{lcccc}
\hline I203 flights & Linear & Mixed (spline) & Curved (pchip) & Curved (spline) \\
\hline Mean absolute lateral deviation $(\mathrm{m})$ & 74.3 & 57.3 & 100.0 & 138.6 \\
Mean STD lateral deviation $(\mathrm{m})$ & 206.2 & 157.2 & 240.6 & 342.4 \\
Mean absolute longitudinal deviation $(\mathrm{m})$ & 102.7 & 101.6 & 97.0 & 152.1 \\
Mean STD longitudinal deviation $(\mathrm{m})$ & 198.6 & 199.8 & 196.4 & 333.5 \\
Mean absolute vertical deviation $(\mathrm{ft})$ & 48.1 & 47.6 & 54.3 & 70.3 \\
Mean STD vertical deviation (ft) & 74.1 & 73.4 & 84.4 & 110.2 \\
\hline
\end{tabular}

STD: standard deviation. 
Table 7. Lateral, longitudinal and vertical deviations (4 days traffic sample).

\begin{tabular}{lllll}
\hline 5069 flights & Linear & Mixed (spline) & Curved (pchip) & Curved (spline) \\
\hline Mean absolute lateral deviation $(\mathrm{m})$ & 50.8 & $33.9(-34 \%)$ & $70.0(38 \%)$ & $94.9(86 \%)$ \\
Mean absolute longitudinal deviation $(\mathrm{m})$ & 69.0 & $67.5(-2 \%)$ & $64.2(-7 \%)$ & $85.8(24 \%)$ \\
Mean absolute vertical deviation $(\mathrm{ft})$ & 46.6 & $46.0(-1 \%)$ & $52.0(11 \%)$ & $66.4(42 \%)$ \\
\hline
\end{tabular}

Table 8. Mean absolute lateral deviation (m).

\begin{tabular}{llllllllrr}
\hline Window size & 3 & 5 & 7 & 9 & 11 & 15 & 20 & 25 & 30 \\
\hline Linear segmentation & 42.2 & 49.6 & 65.5 & 83.5 & 96 & 126.5 & 165.8 & 202.5 & 229.4 \\
Mixed segmentation & 33.2 & 32.6 & 35 & 40.6 & 37 & 49.4 & 62.4 & 75.7 & 88.9 \\
\hline
\end{tabular}

Table 9. Mean absolute longitudinal deviation (m).

\begin{tabular}{llllllllll}
\hline Window size & 3 & 5 & 7 & 9 & 11 & 15 & 20 & 25 & 30 \\
\hline Linear segmentation & 74.7 & 62.9 & 63.4 & 72.5 & 82.4 & 110.7 & 144.1 & 168.7 & 183.4 \\
Mixed segmentation & 73.4 & 61.8 & 59.6 & 69.7 & 70.8 & 93.2 & 110.7 & 125 & 138.7 \\
\hline
\end{tabular}

Table 10. Mean absolute vertical deviation ( $\mathrm{ft}$ ).

\begin{tabular}{llllllllll}
\hline Window size & 3 & 5 & 7 & 9 & 11 & 15 & 20 & 25 & 30 \\
\hline Linear segmentation & 51.7 & 47.1 & 45.1 & 46.4 & 49.7 & 56.8 & 66.3 & 73.9 & 76.7 \\
Mixed segmentation & 51.3 & 46.5 & 44.3 & 45.5 & 48.8 & 56.5 & 66.8 & 75.3 & 79.1 \\
\hline
\end{tabular}

several metres below (see Table 8). The lateral deviation presents a minimum for the mixed segmentation between 3 and 7 points size.

Table 9 indicates that the longitudinal deviations are lower for the different window sizes with mixed segmentation and for both segmentations the curve deviations have a minimum error for sizes having between 5 and 7 points.

As mentioned before the mean absolute vertical error is very similar for both segmentations and it has a minimum for a 7 points window (see Table 10).

\section{Conclusions}

The development of a method to model the trajectories of airplanes in high density terminal areas based on radar tracks has to take into account the frequent changes in heading, speed and flight level of the planes to interpolate adequately the tracks.

Four segmentation methods have been analysed over a huge number of radar tracks: linear, mixed (linear segments for straight lines and cubic spline functions for curves), curved with cubic spline functions and curved with shape preserving cubic spline functions. The minimum errors between the interpolated and the actual track are obtained with the mixed segmentation, but they are quite similar in the vertical profile.

Therefore, the model to interpolate flight radar tracks in a TMA with the best adjustment is mixed (linear-spline) segmentation in the horizontal plane and linear segmentation in the vertical profile to simplify the algorithms. This model will provide average errors between the actual and segmented track of less than $100 \mathrm{~m}$ (lateral $34 \mathrm{~m}$, longitudinal $67 \mathrm{~m}$ and vertical $47 \mathrm{ft}$ ) which is appropriated in a terminal area where the minimum standard separation can be 3 nautical miles.

The sensitivity analysis about the window size for the segmentation process shows that the errors between the segmented and the actual track have a minimum around 3-7 points of size depending on the type of error, being the optimum window size 5 points. A segmentation process with a window of more than 5 points cannot detect some of the changes in the flight, so that the errors will grow with the size window.

The segmentation model is applicable to the development of a 3D CRM using flight radar tracks for a high density TMA. The 3D CRM might support to an air navigation service provider to assess the safety level in this kind of airspaces. 


\section{Funding}

This research received no specific grant from any funding agency in the public, commercial, or not-for-profit sectors.

\section{Conflict of interest}

None declared.

\section{References}

1. ICAO, All-Weather Operations Panel: Report on the 15th meeting, AWOP/15-WP/718, 1994.

2. CANSO. Safety performance measurement benchmarking report, v5.0. 2013.

3. EUROCONTROL, PRC performance review report 2013, 2014.

4. Marks BL. Air traffic control separation standards and collision risk, royal aircraft establishment, Tech. Note No. Math. 91, 1963.

5. Reich PG. Analysis of long range air traffic systems separation standards I. II \& III. I Inst Novig 1966; 19: 88169331-98186347.

6. ICAO, Report of the review of the general concept of separation panel. In: Sixth meeting, Montreal. Canada. ICAO, 28 November-15 December 1988.

7. Bakker GJ and Blom HAP. Air traffic collision risk modelling, NLR. In: Procedures 32nd IEEE conference on decision and control, San Antonio, TX, 1993, pp.1464-1469. Piscataway, NJ: IEEE.

8. Brooker P. Radar inaccuracies and mid-air collision risk: Part 2 en route radar separation minima. $J$ Navig $2004 ; 57 ; 39-51$.

9. Carpenter BD and Kuchar JK. Probability-based collision alerting logic for closely-spaced parallel approach. In: 35th Aerospace sciences meeting and exhibit, Reno, NV, 1997, AIAA paper 1997-222 Reston. VA: AIAA.

10. Powell JD and Sharon H. Assessment of the possibility of a midair collision during an ultra closely spaced parallel approach. In: $A I A A$ guidance, navigation, and control conference and exhibit, Montreal, Canada, 2001, AIAA paper 2001-4205. Reston, VA: AIAA.

11. Kuchar JK and Winder LF. Generalized philosophy of alerting with applications to parallel approach collision prevention. In: AIAA guidance, navigation, and control conference and exhibit. Montreal, Canada, 2001. AIAA paper 2001-4052. Reston, VA: AIAA.
12. Winder LF and Kuchar JK. Evaluation of collision avoidance maneuvers for parallel approach. $J$ Guid Control Dyn 1999; 22: 801-807.

13. Powell JD. Assessment of the possibility of a midair collision during an ultra closely spaced parallel approach. In: AIAA guidance, navigation, and control conference and exhibit, Montreal, Canada, 2001. AIAA paper 2001-4205. Reston, VA: AIAA.

14. Shepherd $R$, Cassell $R$, Rannoch $T$, et al. A reduced aircraft separation risk assessment model. In: $A I A A$ guidance, navigation, and control conference, New Orleans. LA, Collection of Technical Papers, 1997. Pt. 3 (A97-37001 10-63), AIAA paper 1997-3735. Reston, VA: AIAA.

15. Garcia E. Development of a 3-dimensional mathenatical collision risk model based on recorded aircraft trajectories to estimate the safety level in high density en-route. airspaces. PhD Thesis. Spain: Polytechnical University of Madrid, 2013.

16. Sáez F, Arnaldo R, Garcia E, et al. Development of a three-dimensional collision risk model tool to assess safety in high density en-route airspaces. Proc IMechE, Part G: J Aerospace Engineening 2010; 224: 1119-1129.

17. Burt L. 3-D Mathematical Model for ECAC Upper Airspace, EUROCONTROL, Final Report, 2000.

18. EUROCONTROL, The EUR RVSM Mathematical Supplement, 2001, MDG 21 DP 01.

19. Garcia $E$ and Sáez F. Development of analytical tools based on the application of the 3-dimensional mathematical collision risk model to radar data. Eurocontrol. 2006, MDG/35DP/10.

20. Garcia E, Sáez F and Izquierdo I. Identification and analysis of proximate events in high density enroute airspaces. In: $7 t h$ USA/Europe ATM R\&D seminar. Barcelona. Spain, 2-5 July 2007 (accessed 5 July 2009). Available at: http://www.atmseminar.org.

21. Burt L. Results of turn identification applied to Maastricht radar data for 3-D collision risk modelling, Eurocontrol, 2005, MDG/31 DP/08.

22. De Boor C. A practical guide to splines. Applied mathematical sciences, vol. 27. New York-Berlin: Springer-Verlag. 2001. 